\title{
C. Caractéristiques des flux financiers et commerciaux entre la Suisse et les pays en développement
}

Gérard Perroulaz et Xavier Tschumi Canosa

\section{(2) OpenEdition Journals}

Édition électronique

URL : http://journals.openedition.org/aspd/328

DOI : 10.4000/aspd.328

ISSN : 1663-9669

Éditeur

Institut de hautes études internationales et du développement

Édition imprimée

Date de publication : 1 avril 2006

Pagination : 235-256

ISBN : 2-88247-061-4

ISSN : $1660-5934$

Référence électronique

Gérard Perroulaz et Xavier Tschumi Canosa, « C. Caractéristiques des flux financiers et commerciaux entre la Suisse et les pays en développement ", Annuaire suisse de politique de développement [En ligne], 25-1 | 2006, mis en ligne le 17 février 2010, consulté le 08 septembre 2020. URL : http:// journals.openedition.org/aspd/328; DOI : https://doi.org/10.4000/aspd.328 


\section{Caractéristiques des flux financiers et commerciaux entre la Suisse et les pays en développement}

\section{C.1. Faits saillants}

Les tableaux introductifs 1 à 3 de la section B donnent la vue d'ensemble des flux financiers et commerciaux entre la Suisse et les pays en développement et en transition (voir la liste des pays selon la classification du Comité d'aide au développement $[\mathrm{CAD}]$ à la fin de cet Annuaire) et mettent en lumière quelques caractéristiques de ces flux:

$\square$ Les apports financiers du secteur privé aux pays en transition sont beaucoup plus importants que les apports d'aide publique et d'aide privée des ONG. Les pays en transition ont en effet reçu 82,1 millions de francs d'aide publique et 14,8 millions de francs de la part des ONG en 2004, alors que les investissements reçus se sont élevés à 10,3 milliards de francs. Pour les pays en développement, le désinvestissement de la Suisse (2,6 milliards de francs) en 2004 a été largement supérieur aux apports de 1'APD (1,5 milliard) et de l'aide privée des ONG (322 millions).

$\square$ Les flux financiers nets entre les pays en développement et la Suisse sont généralement en faveur de cette dernière. Si l'on prend en considération uniquement les flux d'aide publique et privée, les investissements et le solde commercial avec certaines régions en développement, on remarque que les flux de ces régions vers la Suisse sont supérieurs aux flux d'aide de la Suisse vers ces régions. Ces données sont partielles, car on ne tient pas compte de certains autres flux, difficiles à répartir par catégories de pays, comme les flux bancaires (service et remboursement de la dette, placements nets dans les banques suisses) ou les versements des migrants en Suisse à leurs familles.

- La répartition géographique est très différente entre l'aide publique (au développement ou non) et les flux d'investissements privés: l'APD et l'aide des ONG sont essentiellement destinées aux pays les moins avancés ou aux autres pays à faible revenu, tandis que les investissements se dirigent surtout vers les pays à revenu moyen et les pays d'Europe centrale, et les exportations vers les pays à revenu moyen, les pays d'Europe centrale et orientale et les pays en développement plus avancés.

On peut relever que certaines régions (comme l'Afrique subsaharienne) et certains groupes de pays (les PMA) restent très dépendants de l'apport de l'aide publique au développement et sont largement à l'écart des relations économiques (investissements et commerce). 


\section{C.1.1. Relations de la Suisse avec l'Afrique subsaharienne}

L'Afrique subsaharienne ${ }^{1}$ représente une part importante de l'aide suisse au développement mais une part très faible des relations économiques du secteur privé suisse.

21,1\% de l'APD bilatérale de la Suisse a été versée pour financer des projets et programmes de développement dans l'Afrique subsaharienne, soit 310,8 millions de francs. 33,5\% des projets financés par les ONG suisses ont concerné l'Afrique subsaharienne, soit 107,9 millions de francs en 2004. L'aide publique et celle des ONG se dirigent surtout vers les pays les plus pauvres de cette région du continent africain.

La même année 2004, les pays africains du sud du Sahara ont versé 2,1 millions de francs au titre du remboursement de la Garantie contre les risques à l'exportation (GRE) et le désinvestissement net vis-à-vis de la Suisse s'élevait à 363 millions de francs. Les flux d'investissement négatifs (désinvestissement) signifient que les rapatriements de bénéfices et les reventes de filiales sont supérieurs au flux de nouveaux investissements (augmentation de la participation au capital dans des filiales, ou création ou achat de nouvelles filiales). Les flux d'investissement de la Suisse vers le continent africain sont négatifs depuis 2001.

Pour avoir une image exacte des flux financiers entre l'Afrique subsaharienne et la Suisse, il faudrait compléter ces données avec des statistiques non quantifiables actuellement de manière détaillées (pays par pays). Parmi les apports destinés à l'Afrique subsaharienne, il faudrait encore tenir compte: $1^{\circ}$ de la part de l'aide multilatérale pour des projets en Afrique (Banque africaine de développement, mais aussi Organisation mondiale de la santé [OMS] et Fonds des nations unies pour l'enfance [UNICEF] par exemple); $2^{\circ}$ des versements des migrants d'origine africaine à leurs familles; $3^{\circ}$ des investissements de portefeuille (globalement deux fois plus élevés que les investissements directs à l'étranger). Dans les flux de l'Afrique subsaharienne vers la Suisse, on ne connait pas l'ampleur des fuites de capitaux et de l'évasion fiscale.

Les importations de la Suisse en provenance de pays d'Afrique subsaharienne se sont élevées à 1,76 milliard de francs en 2004, ce qui représente à peine 1,3\% des importations totales de la Suisse. $90 \%$ des importations en provenance de l'Afrique subsaharienne proviennent de l'Afrique du Sud (1 milliard de francs importés en 2004, métaux précieux) et du Nigeria (576 millions, pétrole).

Les exportations de la Suisse à destination de pays d'Afrique subsaharienne se sont élevées à 1,16 milliard de francs en 2004 , ce qui représente $0,8 \%$ des exportations totales de la Suisse. Près de la moitié de ces exportations se dirigent vers l'Afrique du Sud (552 millions). Le solde commercial (exportations moins importations) de la Suisse envers l'Afrique subsaharienne est donc déficitaire de près de 600 millions de francs. Cela s'explique surtout par les importations de pétrole du Nigeria et par les importations de métaux précieux d'Afrique du Sud.

L'engagement total de la GRE pour couvrir des exportations vers l'Afrique sub-

Tous les pays du continent africain sauf le Maroc, l'Algérie, la Tunisie, la Libye et l'Egypte. 
saharienne s'élevait à 174 millions de francs fin 2004, soit 2\% des engagements totaux de la GRE. Dans son rapport annuel 2004, le Bureau de la GRE reconnaît que cet instrument ne peut souvent pas assurer le risque d'un engagement en Afrique (y compris cette fois l'Afrique du Nord), un marché marginal pour l'industrie d'exportation. Ces pays restent donc dépendants des fonds d'aide au développement.

\section{C.1.2. Relations de la Suisse avec les pays les moins avancés (PMA)}

La situation des 49 pays les moins avancés (PMA) est semblable à celle des pays d'Afrique subsaharienne: alors que les PMA ont reçu de la Suisse en 2004 22\% de son APD totale et $43 \%$ de l'aide des ONG, seul 1,8\% des importations en provenance des pays en développement et seuls $2 \%$ des exportations vers les pays en développement concernaient les PMA. Ces derniers ne représentent que 0,12\% des importations totales de la Suisse et $0,24 \%$ de ses exportations totales.

\section{C.2. Commerce extérieur de la Suisse}

\section{C.2.1. Vue d'ensemble}

Schéma 3: Commerce de la Suisse avec les pays en développement et en transition, 2004 (en millions de francs)

Source: adaptée de: Aus Fehlern lernen? Die Schweiz und die Dritte Welt, Richard Gerster, Edition Ex Libris, Zurich, p. 65;

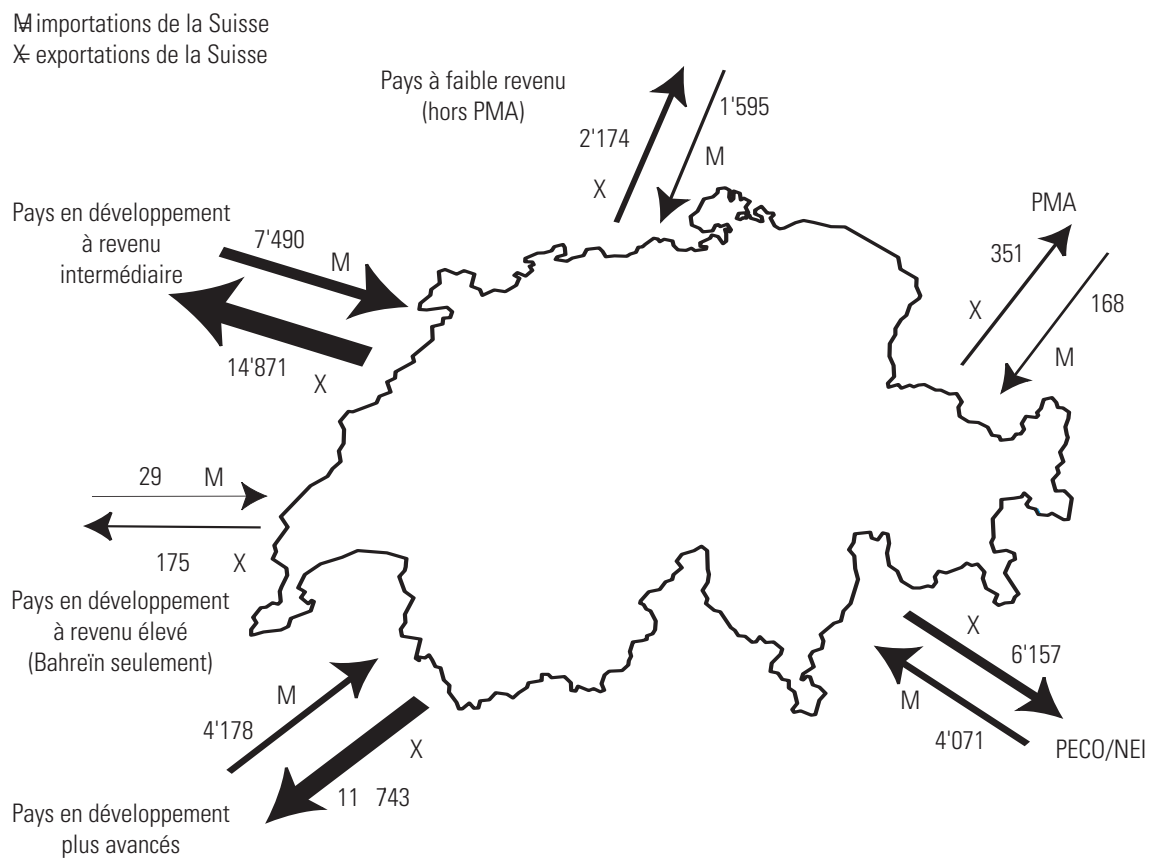

Direction générale des douanes, Commerce extérieur de la Suisse. Statistique selon les marchandises et les pays, janvierdécembre 2004, Berne 2005, pp. 869-871 pour les chiffres actualisés.

$\square$ Solde de la balance commerciale (exportations moins importations) 
D'une manière générale, la Suisse a une balance commerciale excédentaire avec la majorité des pays en développement et des pays en transition, alors que sa balance commerciale est plutôt déficitaire avec les pays industrialisés (voir les trois dernières colonnes des tableaux de la section B). Par exemple, en 2004, la Suisse a importé pour 45,5 milliards de francs de produits en provenance d'Allemagne, son partenaire commercial le plus important, et exporté pour 29,8 milliards de francs vers ce pays, soit un déficit commercial de 15,7 milliards de francs. L'excédent commercial avec l'ensemble des pays en développement s'est élevé en 2004 à 8,3 milliards de francs; l'excédent commercial avec les pays en développement avancés a atteint quant à lui 7,3 milliards de francs, et celui avec les pays d'Europe centrale et orientale 2,1 milliards de francs. Le cas de la Chine est intéressant à relever, car la Suisse importait ces dernières années beaucoup plus qu'elle n'exportait vers ce pays, avec un solde commercial négatif allant en diminuant. Le solde commercial est devenu légèrement positif dès 2003. Le graphique 1 montre la liste des pays en développement ou en transition qui représentent des marchés particulièrement importants pour la Suisse.

\section{Graphique 1: Pays en développement et en transition avec lesquels l'excédent commercial est le plus important, 2004 (en millions de francs)}

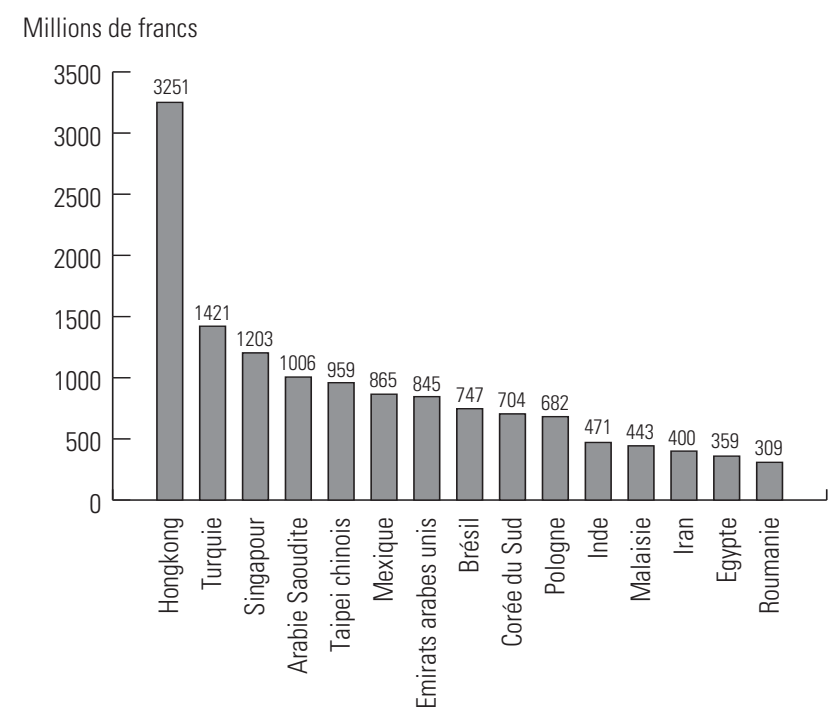

Source: Direction générale des douanes, Commerce extérieur de la Suisse. Statistique selon les marchandises et les pays, janvier-décembre 2004, Berne 2005, pp. 872-877. 
La Suisse entretient des relations commerciales surtout avec les pays voisins et les pays industrialisés européens et d'outre-mer (Etats-Unis, Canada et Japon). Comme on le voit dans le tableau 4, 10 pays en développement ou en transition figurent parmi les 25 principaux pays fournisseurs de la Suisse. Ils sont 11 dans les 25 principaux pays clients de la Suisse.

Tableau 5: Les 25 premiers partenaires commerciaux de la Suisse, 2004

\begin{tabular}{|c|c|c|c|c|c|c|c|c|}
\hline \multicolumn{4}{|c|}{ Principaux fournisseurs } & \multicolumn{5}{|c|}{ Principaux clients } \\
\hline \multicolumn{4}{|c|}{ Importations } & \multicolumn{5}{|c|}{ Exportations } \\
\hline Pays $^{a}$ & Mio fr. & $\%$ & $\sum \%$ & & Pays $^{a}$ & Mio fr. & $\%$ & $\sum \%$ \\
\hline 1. Allemagne féd. & $45^{\prime} 484.3$ & 32.8 & 32.8 & 1. & Allemagne féd. & $29^{\prime} 759.6$ & 20.2 & 20.2 \\
\hline 2. Italie & $15^{\prime} 616.0$ & 11.3 & 44.0 & 2. & Etats-Unis & $15^{\prime} 316.9$ & 10.4 & 30.6 \\
\hline 3. France & $13^{\prime} 711.3$ & 9.9 & 53.9 & 3. & France & $12^{\prime} 825.1$ & 8.7 & 39.3 \\
\hline 4. Pays-Bas & 6'906.2 & 5.0 & 58.9 & & Italie & $12^{\prime} 254.5$ & 8.3 & 47.6 \\
\hline 5. Etats-Unis & 6’583.9 & 4.7 & 63.6 & 5. & Grande-Bretagne & 7'505.9 & 5.1 & 52.7 \\
\hline 6. Autriche & $5^{\prime} 930.9$ & 4.3 & 67.9 & 6. & Espagne & $5^{\prime} 923.8$ & 4.0 & 56.7 \\
\hline 7. Grande-Bretagne & $5 \prime 549.8$ & 4.0 & 71.9 & 7. & Japon & $5 ' 717.7$ & 3.9 & 60.6 \\
\hline 8. Irlande & 4'691.0 & 3.4 & 75.3 & 8. & Autriche & 4'774.3 & 3.2 & 63.8 \\
\hline 9. Belgique & 4'170.0 & 3.0 & 78.3 & 9. & Pays-Bas & 4'390.9 & 3.0 & 66.8 \\
\hline 10. Espagne & $3^{\prime} 401.3$ & 2.5 & 80.7 & 10. & Hongkong & 4'072.7 & 2.8 & 69.6 \\
\hline 11. Japon & 2'930.5 & 2.1 & 82.8 & 11. & Chine & $3{ }^{\prime} 075.7$ & 2.1 & 71.7 \\
\hline 12. Chine & $2^{\prime} 827.1$ & 2.0 & 84.9 & 12. & Belgique & $2 ' 763.2$ & 1.9 & 73.5 \\
\hline 13. Suède & 1'658.2 & 1.2 & 86.1 & 13. & Canada & 1'986.1 & 1.3 & 74.9 \\
\hline 14. Danemark & 1'100.5 & 0.8 & 86.9 & 14. & Turquie & 1'933.9 & 1.3 & 76.2 \\
\hline 15. Afrique du Sud & 1'012.0 & 0.7 & 87.6 & 15. & Suède & 1'645.0 & 1.1 & 77.3 \\
\hline 16. Russie & 1'005.0 & 0.7 & 88.3 & 16. & Singapour & 1'496.7 & 1.0 & 78.3 \\
\hline 17. Rép. tchèque & 981.2 & 0.7 & 89.0 & 17. & Taïpei chinois & 1'489.2 & 1.0 & 79.3 \\
\hline 18. Finlande & 859.5 & 0.6 & 89.7 & 18. & Pologne & 1'336.3 & 0.9 & 80.2 \\
\hline 19. Hongkong & 821.5 & 0.6 & 90.2 & 19. & Corée du Sud & 1'309.7 & 0.9 & 81.1 \\
\hline 20. Libye & 790.1 & 0.6 & 90.8 & 20. & Australie & 1'282.9 & 0.9 & 82.0 \\
\hline 21. Hongrie & 769.3 & 0.6 & 91.4 & 21. & Brésil & 1'249.7 & 0.8 & 82.8 \\
\hline 22. Pologne & 654.8 & 0.5 & 91.8 & 22. & Russie & 1'211.2 & 0.8 & 83.7 \\
\hline 23. Canada & 654.4 & 0.5 & 92.3 & 23. & Grèce & 1'180.7 & 0.8 & 84.5 \\
\hline 24. Thaïlande & 646.5 & 0.5 & 92.8 & 24. & Arabie saoudite & 1'082.8 & 0.7 & 85.2 \\
\hline 25. Corée du Sud & 605.5 & 0.4 & 93.2 & 25. & Emirats arabes unis & 1'080.8 & 0.7 & 85.9 \\
\hline Autres pays & $9^{\prime} 417.7$ & 6.8 & 100.0 & & Autres pays & $20^{\prime} 723.3$ & 14.1 & 100.0 \\
\hline Total & $138 ' 778.4$ & & 100.0 & & Total & 147 '388.4 & & 100.0 \\
\hline
\end{tabular}

Source: Direction générale des douanes, Commerce extérieur de la Suisse, Statistique selon les marchandises et les pays janvier-décembre 2004, 2005, pp. 872-877.

a En gras, pays compris dans la liste des pays en développement (partie I de la liste du CAD) ou des pays en transition (partie II de la liste du CAD). 


\section{C.2.2. Commerce de la Suisse avec les pays en développement et pays en transition}

Les tableaux 6 et 7 montrent les 25 pays principaux partenaires commerciaux, respectivement dans le groupe des pays en développement et dans le groupe des pays en transition (selon la classification des pays établie par le CAD).

Tableau 6: Les 25 premiers partenaires commerciaux de la Suisse dans les pays en développementa ${ }^{a}, 2004$ (en millions de francs et en pourcentage)

\begin{tabular}{|c|c|c|c|c|c|c|c|}
\hline \multicolumn{4}{|c|}{ Principaux fournisseurs } & \multicolumn{4}{|c|}{ Principaux clients } \\
\hline \multicolumn{4}{|c|}{ Importations } & \multicolumn{4}{|c|}{ Exportations } \\
\hline Pays & Mio fr. & $\%^{\mathrm{b}}$ & $\sum \%^{\mathrm{c}}$ & Pays & Mio fr. & $\%^{\mathrm{b}}$ & $\sum \%^{\mathrm{c}}$ \\
\hline 1. Chine & $2^{\prime} 827.1$ & 30.5 & 30.5 & 1. Chine & $3^{\prime} 075.7$ & 17.5 & 17.5 \\
\hline 2. Afrique du Sud & 1'012.0 & 10.9 & 41.4 & 2. Turquie & 1'933.9 & 11.0 & 28.5 \\
\hline 3. Thaïlande & 646.5 & 7.0 & 48.3 & 3. Brésil & 1'249.7 & 7.1 & 35.6 \\
\hline 4. Nigeria & 576.4 & 6.2 & 54.5 & 4. Arabie saoudite & 1'082.8 & 6.2 & 41.8 \\
\hline 5. Inde & 548.1 & 5.9 & 60.4 & 5. Mexique & $11^{\prime} 044.1$ & 5.9 & 47.7 \\
\hline 6. Turquie & 512.6 & 5.5 & 66.0 & 6. Inde & 1'019.1 & 5.8 & 53.5 \\
\hline 7. Brésil & 503.1 & 5.4 & 71.4 & 7. Thaïlande & 815.0 & 4.6 & 58.2 \\
\hline 8. Malaisie & 184.4 & 2.0 & 73.4 & 8. Malaisie & 627.6 & 3.6 & 61.7 \\
\hline 9. Iran & 184.2 & 2.0 & 75.4 & 9. Iran & 584.2 & 3.3 & 65.1 \\
\hline 10. Mexique & 178.8 & 1.9 & 77.3 & 10. Afrique du Sud & 552.2 & 3.1 & 68.2 \\
\hline 11. Liban & 178.2 & 1.9 & 79.2 & 11. Egypte & 391.5 & 2.2 & 70.4 \\
\hline 12. Vietnam & 171.0 & 1.8 & 81.1 & 12. Pakistan & 340.2 & 1.9 & 72.4 \\
\hline 13. Algérie & 168.8 & 1.8 & 82.9 & 13. Indonésie & 286.4 & 1.6 & 74.0 \\
\hline 14. Indonésie & 157.3 & 1.7 & 84.6 & 14. Argentine & 257.2 & 1.5 & 75.5 \\
\hline 15. Maroc & 105.4 & 1.1 & 85.7 & 15. Croatie & 225.2 & 1.3 & 76.7 \\
\hline 16. Kazakhstan & 104.5 & 1.1 & 86.8 & 16. Philippines & 216.8 & 1.2 & 78.0 \\
\hline 17. Philippines & 91.9 & 1.0 & 87.8 & 17. Serbie-et-Monténégro & 215.5 & 1.2 & 79.2 \\
\hline 18. Bangladesh & 77.4 & 0.8 & 88.7 & 18. Colombie & 209.8 & 1.2 & 80.4 \\
\hline 19. Arabie saoudite & 77.0 & 0.8 & 89.5 & 19. Panama & 193.5 & 1.1 & 81.5 \\
\hline 20. Colombie & 68.0 & 0.7 & 90.2 & 20. Algérie & 191.8 & 1.1 & 82.6 \\
\hline 21. Costa Rica & 64.4 & 0.7 & 90.9 & 21. Liban & 178.8 & 1.0 & 83.6 \\
\hline 22. Croatie & 62.0 & 0.7 & 91.6 & 22. Bahreïn & 174.8 & 1.0 & 84.6 \\
\hline 23. Chili & 58.1 & 0.6 & 92.2 & 23. Maroc & 167.8 & 1.0 & 85.6 \\
\hline 24. Sri Lanka & 54.6 & 0.6 & 92.8 & 24. Nigeria & 141.8 & 0.8 & 86.4 \\
\hline 25. Argentine & 48.6 & 0.5 & 93.3 & 25. Chili & 135.5 & 0.8 & 87.1 \\
\hline $\begin{array}{l}\text { Autres pays en } \\
\text { développement }\end{array}$ & 620.2 & 6.7 & 100.0 & $\begin{array}{l}\text { Autres pays en } \\
\text { développement }\end{array}$ & $2^{\prime} 260.1$ & 12.9 & 100.0 \\
\hline Total & $9^{\prime} 280.6$ & & 100.0 & Total & $17 ' 570.9$ & & 100.0 \\
\hline
\end{tabular}

Source: Direction générale des douanes, Commerce extérieur de la Suisse. Statistique selon les marchandises et les pays, janvier-décembre 2004, Berne 2005, pp. 872-877.

a Pays en développement selon la partie I de la liste du CAD (reproduite à la fin de l'Annuaire).

b Pourcentage du pays dans le total des importations des pays en développement, respectivement des exportations vers ces pays.

c Pourcentage cumulé des premiers pays dans le total des importations des pays en développement, respectivement des exportations vers ces pays. 
Tableau 7: Les 25 premiers partenaires commerciaux de la Suisse dans les pays en transition ${ }^{\mathrm{a}}, 2004$

\begin{tabular}{|c|c|c|c|c|c|c|c|}
\hline \multicolumn{4}{|c|}{ Principaux fournisseurs } & \multicolumn{4}{|c|}{ Principaux clients } \\
\hline \multicolumn{4}{|c|}{ Importations } & \multicolumn{4}{|c|}{ Exportations } \\
\hline Pays & Mio fr. & $\%^{\mathrm{b}}$ & $\sum \% c$ & Pays & Mio fr. & $\%^{b}$ & $\sum \% c$ \\
\hline 1. Russie & 1'005.0 & 12.2 & 12.2 & 1. Hongkong & 4'072.7 & 23.1 & 23.1 \\
\hline 2. Rép. tchèque & 981.2 & 11.9 & 24.1 & 2. Singapour & 1'496.7 & 8.5 & 31.7 \\
\hline 3. Hongkong & 821.5 & 10.0 & 34.0 & 3. Taïpei chinois & 1'489.2 & 8.5 & 40.1 \\
\hline 4. Libye & 790.1 & 9.6 & 43.6 & 4. Pologne & 1'336.3 & 7.6 & 47.7 \\
\hline 5. Hongrie & 769.3 & 9.3 & 52.9 & 5. Corée du Sud & 1'309.7 & 7.4 & 55.2 \\
\hline 6. Pologne & 654.8 & 7.9 & 60.9 & 6. Russie & 1'211.2 & 6.9 & 62.0 \\
\hline 7. Corée du Sud & 605.5 & 7.3 & 68.2 & 7. Rép. tchèque & 1'148.2 & 6.5 & 68.6 \\
\hline 8. Taïpei chinois & 529.7 & 6.4 & 74.6 & 8. Emirats arabes unis & 1'080.8 & 6.1 & 74.7 \\
\hline 9. Israël & 413.7 & 5.0 & 79.7 & 9. Hongrie & 881.8 & 5.0 & 79.7 \\
\hline 10. Slovaquie & 295.5 & 3.6 & 83.2 & 10. Israël & 659.7 & 3.7 & 83.5 \\
\hline 11. Singapour & 293.9 & 3.6 & 86.8 & 11. Roumanie & 463.5 & 2.6 & 86.1 \\
\hline 12. Emirats arabes unis & 235.5 & 2.9 & 89.7 & 12. Slovénie & 294.9 & 1.7 & 87.8 \\
\hline 13. Slovénie & 200.1 & 2.4 & 92.1 & 13. Slovaquie & 291.0 & 1.7 & 89.4 \\
\hline 14. Vierges, îles & 160.4 & 1.9 & 94.0 & 14. Bulgarie & 282.5 & 1.6 & 91.0 \\
\hline 15. Roumanie & 154.9 & 1.9 & 95.9 & 15. Qatar & 236.5 & 1.3 & 92.4 \\
\hline 16. Bulgarie & 64.9 & 0.8 & 96.7 & 16. Koweit & 234.8 & 1.3 & 93.7 \\
\hline 17. Ukraine & 50.0 & 0.6 & 97.3 & 17. Ukraine & 229.7 & 1.3 & 95.0 \\
\hline 18. Bahamas & 49.2 & 0.6 & 97.9 & 18. Libye & 189.5 & 1.1 & 96.1 \\
\hline 19. Lituanie & 46.0 & 0.6 & 98.4 & 19. Lettonie & 123.4 & 0.7 & 96.8 \\
\hline 20. Gibraltar & 43.4 & 0.5 & 99.0 & 20. Lituanie & 95.0 & 0.5 & 97.3 \\
\hline 21. Estonie & 33.7 & 0.4 & 99.4 & 21. Malte & 85.9 & 0.5 & 97.8 \\
\hline 22. Lettonie & 11.7 & 0.1 & 99.5 & 22. Chypre & 72.1 & 0.4 & 98.2 \\
\hline 23. Aruba & 10.4 & 0.1 & 99.6 & 23. Vierges, îles & 61.0 & 0.3 & 98.6 \\
\hline 24. Macao & 7.3 & 0.1 & 99.7 & 24. Estonie & 50.7 & 0.3 & 98.9 \\
\hline 25. Oatar & 7.3 & 0.1 & 99.8 & 25. Bélarus & 43.8 & 0.2 & 99.1 \\
\hline $\begin{array}{l}\text { Autres pays } \\
\text { en transition }\end{array}$ & 14.5 & 0.2 & 100.0 & $\begin{array}{l}\text { Autres pays } \\
\text { en transition }\end{array}$ & 152.8 & 0.9 & 100.0 \\
\hline Total & 8'249.5 & & 100.0 & Total & $17 ' 593.4$ & & 100.0 \\
\hline
\end{tabular}

Source: Direction générale des douanes, Commerce extérieur de la Suisse. Statistique selon les marchandises et les pays, janvier-décembre 2004, 2005, pp. 872-877.

a Pays en transition selon la partie II de la liste du CAD (reproduite à la fin de l'Annuaire).

b Pourcentage du pays dans le total des importations des pays en transition, respectivement des exportations vers ces pays.

c Pourcentage cumulé des premiers pays dans le total des importations des pays en transition, respectivement des exportations vers ces pays. 


\section{C.3.1. Investissements directs à l'étranger}

Le rôle des investissements directs à l'étranger est important pour les pays en développement: ils favorisent le transfert de technologies et la création d'emplois; en outre, moins volatils que les investissements de portefeuille, ils constituent des capitaux relativement stables pour les pays en développement.

\section{$\square$ Flux d'investissements directs à l'étranger en 2004}

Les flux d'investissements directs à l'étranger émanant de la Suisse se sont élevés à 33,4 milliards de francs en 2004, dont 15,8 milliards dans les pays de l'Union européenne, 8,9 milliards en Amérique du Nord (Canada et EtatsUnis), 1,1 milliard en Australie/Nouvelle Zélande et 1 milliard vers le Japon. Les données ne sont pas tout à fait comparables aux statistiques des années précédentes, puisque le nombre d'entreprises fournissant des données sur leurs investissements est passé de 850 à 1100 , ce qui a gonflé le volume des investissements de $11 \%$.

Les statistiques publiées par la Banque nationale suisse $(\mathrm{BNS})^{2}$ ne permettent pas de voir la part exacte de ces investissements destinés au groupe des pays en développement ou au groupe des pays en transition. La BNS ne fait qu'une répartition par continent des investissements. Les données reprises par la Direction du développement et de la coopération (DDC) permettent de voir la part relativement faible des investissements qui vont vers les pays en développement ou les pays en transition (voir tableaux de la section B de cette partie statistique). Selon les données publiées par la DDC sur la base de la classification des pays du $\mathrm{CAD}$, on constate que les investissements directs suisses à destination du groupe des «pays en développement plus avancés» se sont élevés à 9 milliards de francs en 2004. 1,3 milliard de francs se sont dirigés vers les pays d'Europe centrale et orientale, alors que le flux net d'investissement à destination des pays en développement est négatif (désinvestissement de 2,6 milliards de francs). Ce solde négatif s'explique notamment par des désinvestissements au Mexique, en Afrique du Sud et aux Philippines.

\section{$\square$ Stock cumulés des investissements directs à l'étranger}

Le graphique 2 montre la répartition géographique du stock des investissements directs à l'étranger émanant de la Suisse à la fin de l'année 2004. Les stocks d'investissements directs à l'étranger s'élevaient à 449 milliards de francs fin 2004, et les entreprises suisses occupaient 1,9 million de personnes dans leurs implantations à l'étranger. Plus de la moitié de ces investissements concerne le secteur des services (289 milliards de francs en 2004, dont 121 milliards sont engagés par des sociétés financières et holdings, 87 milliards par des compagnies d'assurances et 55 milliards par des banques suisses).

En 2004, 44,1\% des stocks totaux étaient investis dans des pays de l'Union européenne, 7,1\% dans les autres pays de l'Europe, 19,1\% en Amérique du

2 BNS, L'évolution des investissements directs en 2004, Zurich, décembre 2005, 38 p. 
Nord (Canada et Etats-Unis). 19,1\% du stock des investissements suisses le sont en Amérique centrale et du Sud, 7,7\% en Asie (y compris Japon), 2,3\% en Océanie et 0,6\% seulement en Afrique (voir graphique 2).

\section{Graphique 2: Répartition des stock d'investissements directs à l'étranger par région, fin 2004 (en milliards de francs)}
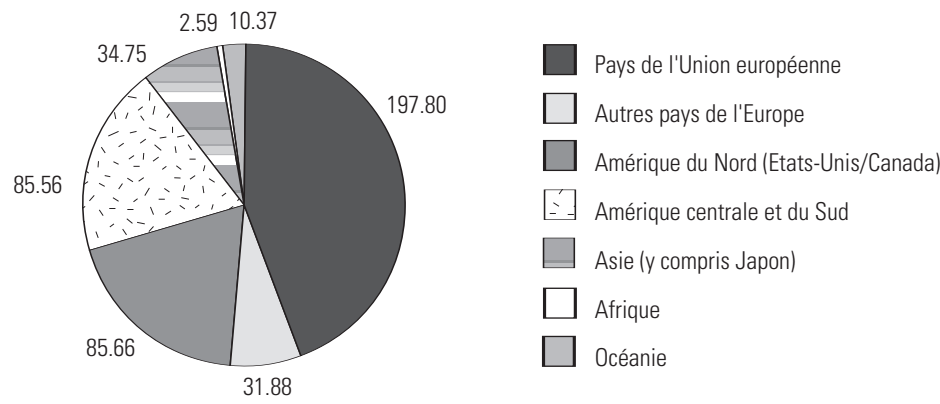

Source: Banque nationale suisse, L'évolution des investissements directs en 2004, Zurich, BNS, décembre 2005, pp 20-21, brochure téléchargeable sur <www.snb.ch $>>$ publications $>$ balance des paiements et position extérieure nette de la Suisse >investissements directs.

Le graphique 3 montre les stocks les plus importants d'investissements directs suisses à l'étranger parmi les pays en développement ou en transition, ainsi que l'évolution du stock de fin 2000 à fin 2004. Une grande partie des investissements dans ces pays concerne les centres financiers offshore des Caraïbes ${ }^{3}$, où le stock total a passé de 31,8 milliards à 67,3 milliards de francs sur la période. Les investissements directs à l'étranger se concentrent sur un petit nombre de pays en développement ou en transition. D'autres pays et régions restent largement à l'écart de ces investissements.

Définitions: La Banque nationale suisse recueille les informations sur les investissements directs à l'étranger lorsqu'un investisseur participe pour au moins $10 \%$ au capital d'une entreprise à l'étranger ou fonde une filiale ou une succursale à l'étranger. Les flux d'investissement enregistrés pendant une année recensent les participations aux fonds propres (fondations, acquisitions, ventes, augmentations de capital), les crédits au sein de groupes ainsi que les bénéfices réinvestis. Ce sont des flux nets, un chiffre négatif signifiant un désinvestissement.

Le stock de fin d'une année additionné du flux net de l'année suivante n'est pas forcément égal au stock à la fin de l'année suivante, car les variations de stocks proviennent aussi de facteurs qui n'apparaissent pas dans les flux (évolution du taux de change, modification dans les méthodes comptables). Les acquisitions financées à l'étranger ne donnent pas toujours lieu non plus à des flux de capitaux. Les statistiques essaient de tenir compte autant que possible du pays du bénéficiaire final (cas des centres financiers offshore par exemple).

3 Centres financiers offshore: Anguilla, Bahamas, Barbade, Bermudes, îles Vierges britanniques, Jamaïque, îles Caïmans, Montserrat, Antilles néerlandaises, Panama, Saint-Kitts-et-Nevis et, depuis 2000, Antigua-et-Barbuda, Belize, Dominique, Grenade, Sainte-Lucie, Saint-Vincent-et-les-Grenadines, îles Turks et Caicos. 


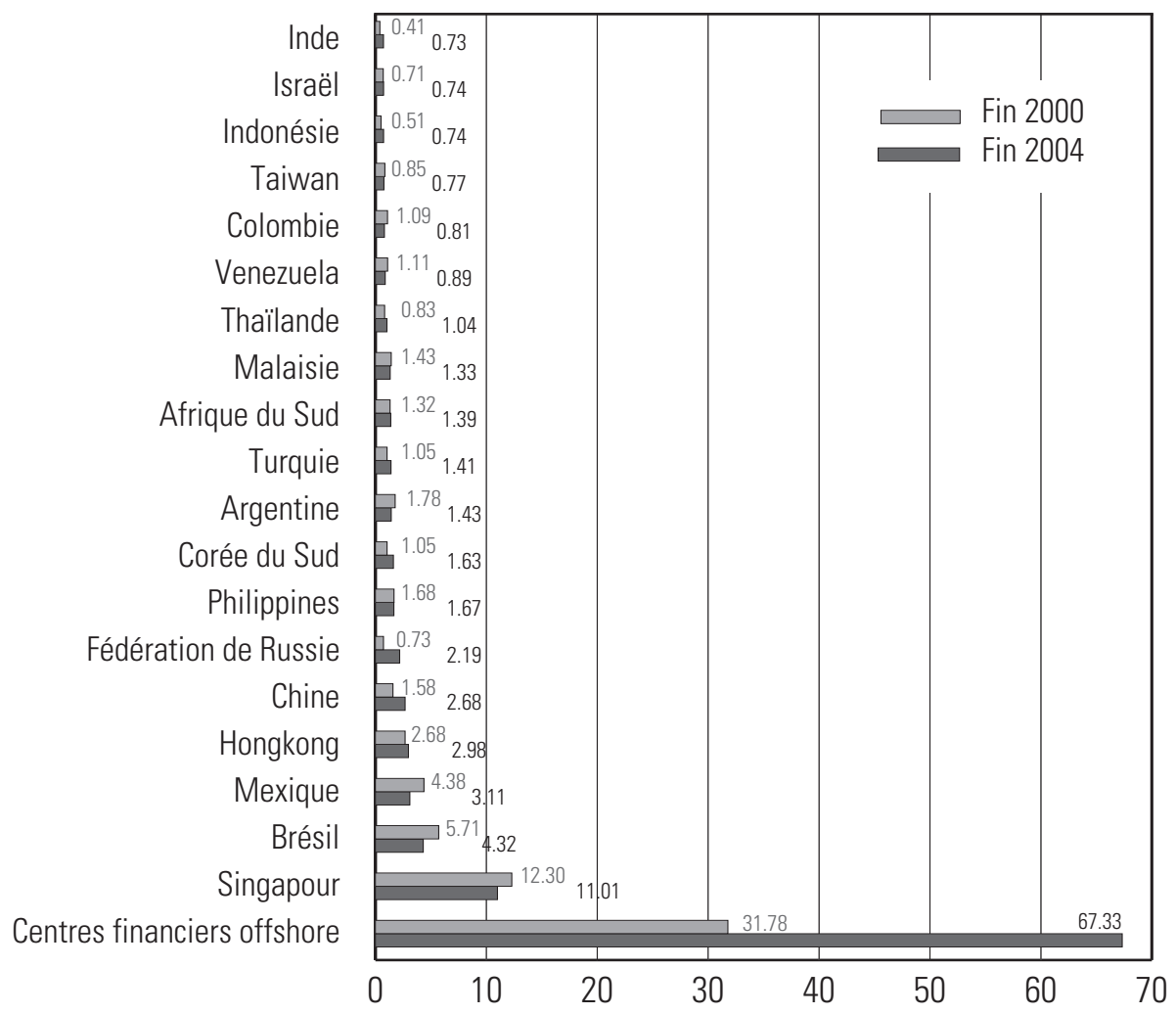

Source: Banque nationale suisse, L'évolution des investissements directs en 2004, Zurich, BNS, décembre 2005, pp. 20-21, brochure téléchargeable sur $<$ www.snb.ch $>>$ publications $>$ balance des paiements et position extérieure nette de la Suisse >investissements directs.

\section{C.3.2. Placements dans les banques suisses}

Les statistiques de la Banque nationale suisse distinguent les opérations que les banques effectuent pour leur propre compte (inscrites au bilan) et les transactions réalisées pour le compte et aux risques des clients (opérations fiduciaires). Cela ne représente qu'une partie des placements financiers car la statistique ne repose que sur les données fournies par 106 banques (sans les données du secteur parabancaire, gestionnaires privés de fortune par exemple).

Les placements totaux de fonds dans la place financière suisse sont estimés à près de 3000 milliards de francs en $2002^{4}$. Selon les chiffres de la Banque nationale suisse pour 106 banques, à la fin 2004 les placements totaux de fonds étrangers dans les banques suisses inscrits au bilan s'élevaient à 1344 milliards

4 Source: Fondation Genève Place financière, <www.geneva-finance.ch > > place financière > place financière de Genève $>$ faits et chiffres. 
de francs et les opérations fiduciaires pour le compte de clients étrangers s'élevaient à 244 milliards de francs.

Le graphique 4 montre les principales provenances des fonds fiduciaires parmi les pays en développement ou en transition. Si une grande partie de ces placements bancaires provient de pays qui ont des ressources économiques importantes (comme les pays producteurs de pétrole par exemple) ou de paradis fiscaux (dont on ne connaît souvent pas l'origine première des fonds), on trouve dans le classement plusieurs pays relativement pauvres, en crise, ou se trouvant dans la liste de Transparency International des pays où règne une corruption endémique.

Graphique 4: Engagements nets à titre fiduciaire des banques établies en Suisse, au 31 décembre 2004 (en millions de francs)

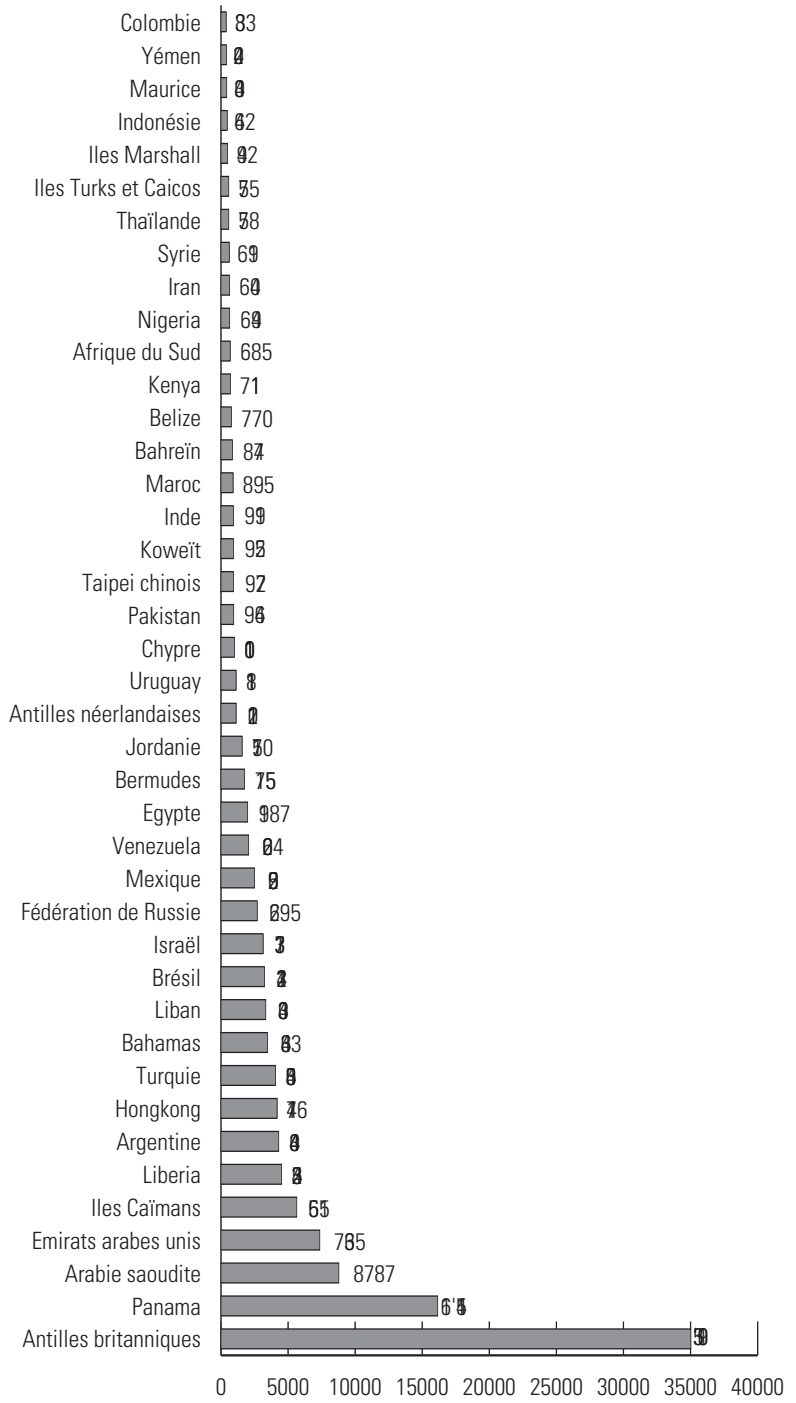

Source: Banque nationale suisse, Les banques suisses 2004, Berne, 2005, à partir des données du tableau 38, "Opérations fiduciaires - répartition géographique», pp. A136-A141, disponible sur <www.snb.ch >>publications $>$ les banques suisses. 


\section{C.4. Aide publique au développement (APD) et aide publique aux pays en transition}

Les graphiques ci-dessous relèvent l'évolution sur vingt ans de l'aide publique au développement et de l'aide privée des ONG.

Après une période de stagnation, voire de baisse, dans les années 1990, l'APD totale des pays membres du CAD a fortement augmenté ces dernières années, en passant de 52,4 milliards de dollars en 2001 à 79,5 milliards de dollars en 2004. Trois facteurs relativisent la forte augmentation de l'APD entre 2003 et 2004 (plus de 10 milliards de dollars):

$\checkmark$ les variations des taux de change des devises des pays membres avec le dollar;

口 les versements importants d'aide à l'Irak (4,7 milliards de dollars en 2004) et à l'Afghanistan (2,2 milliards);

- la comptabilisation dans l'APD de montants importants au titre des réaménagements de dettes de pays en développement (pour un montant total de 7,2 milliards de dollars en 2004).

Exprimée en pourcentage du revenu national brut (RNB), cette hausse est cependant plus modeste, puisque l'APD des pays du CAD est passée de $0,22 \%$ du RNB en 2001 à $0,26 \%$ en 2004, ce qui reste bien en deçà de l'objectif de $0,7 \%$ du RNB fixé par les Nations unies et même du 0,33\% enregistré en moyenne pendant la période 1980-1992. Seuls cinq pays dépassent l'objectif des $0,7 \%$ du RNB, soit la Norvège, le Danemark, le Luxembourg, la Suède et les Pays-Bas.

\section{Graphique 5: "Vingt ans de coopération » - Evolution de l'aide des pays du CAD (aide publique au développement [APD] et aide publique aux pays en transition [AP]), 1985-2004 (en millions de dollars et en pourcentage du RNB)}



Source: OCDE, statistiques du CAD, <www.oecd.org/cad >. 
L'APD de la Suisse a également atteint des niveaux records en 2003 et 2004, dépassant le montant d'APD versé en 1992 lorsque la Suisse avait rejoint les institutions de Bretton Woods (voir graphique 6). L'augmentation de l'APD de la Suisse de 2003 à 2004 s'explique surtout par la décision du Conseil fédéral, en mai 2005, de comptabiliser dans l'APD les montants liés à la présence des requérants d'asile en provenance des pays en développement la première année de leur séjour. Auparavant, la Suisse ne comptait dans l'APD que les frais pour les personnes auxquelles l'asile était accordé, et non pas pour tous les requérants. Le montant comptabilisé dans l'APD est ainsi passé de 48,6 millions de francs en 2003 à 256,1 millions de francs en 2004.

De plus en plus de pays du CAD comptabilisent une partie des frais liés à la présence de réquérants d'asile dans leur $\mathrm{APD}$. Ces montants représentaient au total 2,1 milliards de dollars en 2004 (contre 1,3 en 2001). Les pays du CAD qui comptabilisent les sommes les plus importantes sont la France (544 millions de dollars en 2004), les Etats-Unis (512), la Suisse (194), la Suède (178) et le Canada (177). Certains pays du CAD refusent pour l'instant de comptabiliser ces frais dans l'APD (Royaume-Uni ou Japon par exemple).

\section{Graphique 6: "Vingt ans de coopération 》 - Evolution de l'aide de la Suisse (aide publique au développement [APD] et aide publique aux pays en transition [AP]), 1985-2004 (en millions de francs et en pourcentage du RNB)}

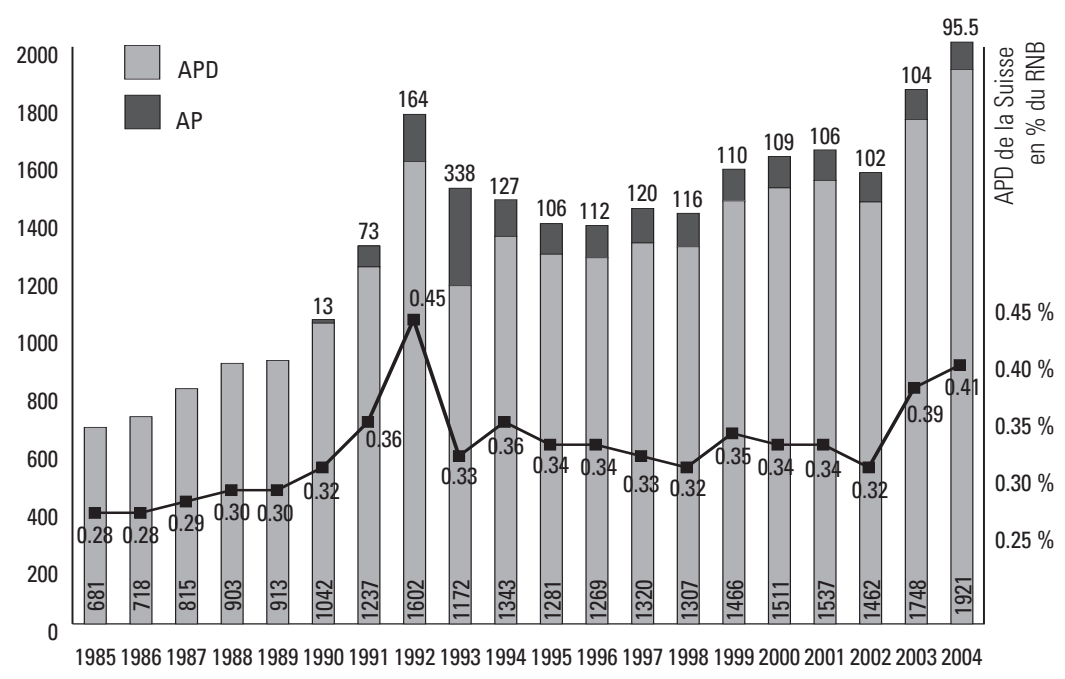

Source: DDC, Aide de la Suisse aux pays en développement et aux pays en transition. Statistiques 2004, Berne, DDC, 2006 (et éditions annuelles précédentes depuis 2001).

Le tableau 8 montre l'évolution de l'aide bilatérale de la Suisse dans les pays d'Europe de l'Est et de la CEI versée par le Secrétariat d'Etat à l'économie (seco) et la DDC entre 1999 et 2004. Ces chiffres ne comprennent pas les versements d'AP des autres offices ou départements fédéraux (Défense, Protection de la population et Sports [DDPS] et Affaires étrangères [DFAE] notamment), ni les versements multilatéraux. Ils ne peuvent donc être comparés aux chiffres des tableaux de la section B de la présente partie statistique. 
Tableau 8: Coopération bilatérale de la Suisse avec les pays d'Europe de l'Est et les pays de la CEI: versements de la DDC et du seco, 1999-2004 (en millions de fr.)

\begin{tabular}{lrrrrrr}
\hline Versements (mio. fr.) & $\mathbf{1 9 9 9}$ & $\mathbf{2 0 0 0}$ & $\mathbf{2 0 0 1}$ & $\mathbf{2 0 0 2}$ & $\mathbf{2 0 0 3}$ & $\mathbf{2 0 0 4}$ \\
\hline Albanie & 10.9 & 9.6 & 8.0 & 13.2 & 13.6 & 8.6 \\
\hline Arménie & & 0.0 & 1.9 & 2.5 & 1.6 & 2.0 \\
\hline Azerbaidjan & 0.8 & 0.8 & 1.1 & 8.3 & 3.4 & 5.3 \\
\hline Bélarus & 0.2 & 0.0 & 0.4 & 0.1 & 0.0 & \\
\hline Bosnie-Herzégovine & 20.9 & 13.6 & 14.1 & 17.9 & 12.7 & 11.5 \\
\hline Bulgarie & 16.7 & 12.2 & 18.5 & 7.9 & 12.7 & 12.9 \\
\hline Croatie & 0.5 & 1.1 & 1.4 & 0.7 & 0.6 & 0.4 \\
\hline Estonie & 0.0 & 0.0 & 0.0 & & 0.0 & 0.0 \\
\hline Géorgie & 0.7 & 1.0 & 1.3 & 2.4 & 3.1 & 3.9 \\
\hline Hongrie & -0.0 & 7.8 & 7.7 & 0.0 & 0.0 & 0.0 \\
\hline Kazakhstan & 0.0 & 0.2 & 0.0 & 0.1 & 0.3 & 0.0 \\
\hline Kirghize, Rép. & 9.0 & 11.1 & 7.5 & 20.8 & 11.0 & 11.9 \\
\hline Kosovo & & 3.1 & 12.0 & 12.1 & 13.8 & 6.2 \\
\hline Lettonie & 4.0 & 1.5 & 1.1 & 0.1 & 0.0 & 0.0 \\
\hline Lituanie & & & & 0.1 & 0.5 & 0.0 \\
\hline Macédoine (ERYM) & 5.8 & 14.0 & 8.7 & 8.3 & 6.0 & 14.7 \\
\hline Moldova & 1.3 & 1.1 & 0.2 & 0.3 & 0.2 & 0.6 \\
\hline Ouzbékistan & 0.9 & 1.3 & 2.0 & 4.5 & 7.6 & 12.2 \\
\hline Pologne & 0.2 & 0.1 & 0.6 & 0.0 & 0.0 & 0.0 \\
\hline Roumanie & 12.2 & 16.1 & 7.1 & 6.6 & 19.6 & 10.5 \\
\hline Russie & 12.4 & 9.1 & 8.7 & 11.3 & 11.0 & 10.1 \\
\hline Serbie-et-Monténégro (sans Kosovo) & 5.6 & 33.5 & 31.4 & 12.7 & 10.0 & 16.4 \\
\hline Slovaquie & 5.2 & 2.7 & 0.0 & 0.1 & 0.7 & 1.6 \\
\hline Slovénie & & & 0.0 & 0.0 & 0.0 & 0.0 \\
\hline Tadjikistan & 0.6 & 6.9 & 9.4 & 6.5 & 17.2 & 20.5 \\
\hline Rép. tchèque & 0.1 & 0.1 & 0.1 & 0.0 & 0.0 & 0.0 \\
\hline Turkménistan & & & & & 1.1 & \\
\hline Ukraine & 18.0 & 14.0 & 2.7 & 5.4 & 6.6 & 6.3 \\
\hline Non ventilé et régional & $\mathbf{3 3 . 0}$ & 54.9 & 51.3 & 45.0 & 47.8 \\
\hline Total & $\mathbf{1 9 4 . 2}$ & $\mathbf{2 0 0 . 9}$ & $\mathbf{1 9 3 . 1}$ & $\mathbf{1 9 8 . 5}$ & $\mathbf{2 0 3 . 6}$ \\
\hline & & & & & &
\end{tabular}

Source: Service statistique de la DDC.

a Du point de vue du droit international, la région du Kosovo est une province de la Serbie-et-Monténégro (ex-République fédérale de Yougoslavie, RFY). En 1999, elle a toutefois été placée sous mandat de I'ONU et a ainsi été soustraite au contrôle de la RFY.

La coopération de la Suisse avec l'Europe de l'Est s'est progressivement déplacée pendant les années 1990 des pays d'Europe centrale (Pologne, Tchéquie, Hongrie, Slovaquie) et de la Baltique (Estonie, Lettonie, Lituanie), devenus membres de l'Union européenne en 2004, vers les Balkans et la CEI. Aujourd'hui, ce sont $70 \%$ de ses moyens qui sont consacrés à l'Europe du Sud-Est et 30\% aux pays de la CEI, principalement dans le Caucase du Sud et en Asie centrale.

Le Conseil fédéral a proposé aux Chambres fédérales deux messages, qui seront traités dans le courant de l'année 2006. Le premier concerne le renouvellement d'un crédit-cadre pour la coopération avec l'Est, qui permettra à la Confédération de poursuivre son engagement en Europe du Sud-Est et auprès des pays de la CEI ; le second concerne la contribution helvétique à la cohésion européenne, qui vise à soutenir financièrement les pays de l'Est nouvellement entrés dans l'Union européenne.

$\mathbb{D}$ Annuaire 2006, $\mathrm{n}^{\circ}$ 1, chap. 3, section 3.1, messages soumis au Parlement: nouvelle loi et nouveau crédit-cadre. 
Le graphique 7 met en évidence la composition diverse des instruments utilisés dans la coopération selon la situation du pays partenaire. La part des instruments de promotion économique dont dispose le seco est importante pour des pays tels que la Chine, le Tadjikistan, le Vietnam et le Mozambique. Les actions de maintien de la paix menées par le DDPS et celles de gestion civile des conflits (DFAE) sont importantes pour des pays comme la Serbie-et-Monténégro. L'aide humanitaire est importante pour un pays comme l'Afghanistan. Les instruments de coopération au développement de la DDC forment une part importante de l'aide en Bolivie, en Tanzanie ou en Inde.

\section{Graphique 7: Aide publique et privée de la Suisse, par catégorie d'aide, 2004 (en milliers de francs)}



Source: DDC, Aide de la Suisse aux pays en développement et aux pays en transition. Statistique 2004, Berne, 2006. 
Tableau 9: Aide des cantons suisses aux pays en développement et aux pays en transition, 2003 et 2004 (en francs)

\begin{tabular}{|c|c|c|c|c|c|c|}
\hline \multirow[b]{2}{*}{ Cantons } & \multicolumn{3}{|c|}{2004} & \multicolumn{3}{|c|}{2003} \\
\hline & APD & AP & CP & APD & AP & CP \\
\hline Argovie & $650 \prime 000$ & - & 105 & $700^{\prime} 000$ & - & 97 \\
\hline Appenzell Rh.-Ext. & $92^{\prime} 168$ & - & 61 & $120^{\prime} 716$ & - & 63 \\
\hline Appenzell Rh.-Int. & - & - & 66 & - & - & 62 \\
\hline Bâle-Campagne & 1'037'900 & $90 \prime 000$ & 116 & $997^{\prime} 000$ & $46^{\prime} 500$ & 120 \\
\hline Bâle-Ville & 1'572'000 & $77^{\prime} 000$ & 166 & 1'577'500 & $225^{\prime} 000$ & 173 \\
\hline Berne & $925^{\prime} 000$ & $185^{\prime} 000$ & 58 & $9766^{\prime} 500$ & $73^{\prime} 000$ & 57 \\
\hline Fribourg & $450 \prime 533$ & $222^{\prime} 980$ & 45 & $17^{\prime} 000$ & - & 51 \\
\hline Genève & $10^{\prime} 040 \prime 942$ & $119^{\prime} 569$ & 155 & 8'044'339 & $306{ }^{\prime} 550$ & 141 \\
\hline Glaris & .. & .. & 77 & .. & .. & 82 \\
\hline Grisons & $82^{\prime} 000$ & $44^{\prime} 000$ & 63 & $82^{\prime} 000$ & $44^{\prime} 000$ & 77 \\
\hline Jura & $400 \prime 000$ & $80 \prime 000$ & 33 & $342 ' 500$ & $100 \prime 000$ & 34 \\
\hline Lucerne & $66^{\prime} 000$ & 4'000 & 63 & $101^{\prime} 000$ & $44^{\prime} 000$ & 67 \\
\hline Neuchâtel & $315^{\prime} 000$ & - & 56 & $305^{\prime} 000$ & - & 55 \\
\hline Nidwald & $99^{\prime} 500$ & $1 ' 000$ & 129 & $70^{\prime} 500$ & $2 \prime 000$ & 129 \\
\hline Obwald & $61^{\prime} 000$ & - & 30 & $27^{\prime} 900$ & - & 35 \\
\hline Schaffhouse & $90 \prime 000$ & $18^{\prime} 000$ & 98 & $90 \prime 000$ & $10^{\prime} 000$ & 107 \\
\hline Schwyz & $83^{\prime} 000$ & - & 117 & 5,000 & - & 112 \\
\hline Soleure & $80^{\prime} 000$ & - & 76 & $30^{\prime} 000$ & $48^{\prime} 000$ & 82 \\
\hline St-Gall & $200^{\prime} 000$ & - & 80 & $390 \prime 000$ & $317^{\prime} 080$ & 80 \\
\hline Tessin & $101^{\prime} 000$ & - & 85 & $90 \prime 000$ & - & 82 \\
\hline Thurgovie & $427^{\prime} 500$ & $21^{\prime} 000$ & 80 & $241^{\prime} 500$ & $13^{\prime} 000$ & 83 \\
\hline Uri & $30 \prime 000$ & $2^{\prime} 000$ & 51 & $31 ' 500$ & 4'016 & 64 \\
\hline Valais & $187^{\prime} 250$ & - & 30 & $52^{\prime} 500$ & - & 30 \\
\hline Vaud & 1'085'112 & $251^{\prime} 049$ & 95 & 1'091'485 & - & 94 \\
\hline Zoug & $550 \prime 000$ & - & 227 & $302^{\prime} 000$ & $20^{\prime} 000$ & 216 \\
\hline Zürich & 2'458'000 & $542^{\prime} 000$ & 157 & $2^{\prime} 800 \prime 000$ & 200 '000 & 160 \\
\hline Total & $20^{\prime} 433^{\prime} 905$ & 1'617'598 & & 17'785'940 & 1'373'146 & \\
\hline
\end{tabular}

$-:$ montant nul.

: montant non disponible.

$\mathrm{APD}=$ Aide publique au développement aux pays et territoires en développement.

$\mathrm{AP}=$ Aide publique aux pays et territoires en transition (pays de l'Europe centrale et orientale, nouveaux Etats indépendants de l'exUnion soviétique, pays et territoires en développement plus avancés).

Source: DDC, Aide de la Suisse aux pays en développement et aux pays en transition, Statistiques 2004, Berne, mars 2006.

$\mathrm{CP}=$ capacité financière des cantons.

L'indice de capacité financière des cantons est fixée par le Conseil fédéral sur la base de quatre coefficients traduisant le revenu cantonal, la capacité et la charge fiscales ainsi que les charges supplémentaires induites par le caractère montagneux des cantons. Cet indice est fixé à 100 pour la Suisse.

Source: Département fédéral des finances (DFF), nouvelle évaluation de la capacité financière des cantons, communiqués de presse du 7 novembre 2001 pour les chiffres 2003 et du 5 novembre 2003 pour les chiffres 2004.

Les chiffres de l'aide publique (au développement) des cantons et des communes sont obtenus par des enquêtes annuelles, dont le taux de réponse est un élément significatif du résultat. Ils doivent donc être considérés comme des minimums. 
Tableau 10: Aide des communes suisses aux pays en développement et aux pays en transition, 2003 et 2004 (en francs)

\begin{tabular}{|c|c|c|c|c|}
\hline \multirow[b]{2}{*}{ Cantons / Communes } & \multicolumn{2}{|c|}{2004} & \multicolumn{2}{|c|}{2003} \\
\hline & APD & AP & APD & AP \\
\hline Argovie & $232 ' 200$ & - & $280^{\prime} 950$ & $5^{\prime} 000$ \\
\hline Ville d'Aarau & $80^{\prime} 000$ & - & $100^{\prime} 000$ & \\
\hline Wettingen & $600^{\prime} 000$ & - & $60^{\prime} 000$ & \\
\hline Baden & - & - & $42^{\prime} 000$ & $5 \times 000$ \\
\hline Appenzell Rh.-Ext. & - & - & $2^{\prime} 300$ & \\
\hline Heiden & - & - & $2^{\prime} 000$ & \\
\hline Bâle-Campagne & $268^{\prime} 874$ & $20^{\prime} 100$ & $233^{\prime} 650$ & $9^{\prime} 000$ \\
\hline Reinach (BL) & $100 ' 874$ & $10^{\prime} 000$ & $85^{\prime} 000$ & \\
\hline Binningen & $78^{\prime} 000$ & $2^{\prime} 000$ & $45^{\prime} 000$ & $5^{\prime} 000$ \\
\hline Bâle-Ville & $241^{\prime} 000$ & $166^{\prime} 000$ & $205^{\prime} 000$ & $170^{\prime} 000$ \\
\hline Riehen & $219^{\prime} 000$ & $166^{\prime} 000$ & $190^{\prime} 000$ & $1700^{\prime} 000$ \\
\hline Berne & $864^{\prime} 796$ & $165^{\prime} 100$ & $691 ' 150$ & $206^{\prime} 760$ \\
\hline Ville de Berne & $355^{\prime} 000$ & - & $240^{\prime} 000$ & \\
\hline Münsingen & $174^{\prime} 300$ & - & $185^{\prime} 600$ & \\
\hline Ittigen & - & $90^{\prime} 000$ & - & 120,000 \\
\hline Thun & $35^{\prime} 000$ & $30^{\prime} 000$ & $30^{\prime} 000$ & \\
\hline Moutier & 12000 & $29^{\prime} 000$ & $2^{\prime} 800$ & $36^{\prime} 000$ \\
\hline Fribourg & $45^{\prime} 500$ & 500 & $33^{\prime} 200$ & 500 \\
\hline Ville de Fribourg & $28^{\prime} 500$ & 500 & $14^{\prime} 000$ & 500 \\
\hline Genève & $6^{\prime} 376^{\prime} 001$ & $205^{\prime} 071$ & $6^{\prime} 252^{\prime} 915$ & $333^{\prime} 781$ \\
\hline Ville de Genève & $3^{\prime} 443^{\prime} 358$ & $89^{\prime} 196$ & $2^{\prime} 881^{\prime} 515$ & $87^{\prime} 675$ \\
\hline Lancy & $540^{\prime} 439$ & $8^{\prime} 000$ & $485^{\prime} 000$ & \\
\hline Carouge (GE) & $412 ' 748$ & - & $360^{\prime} 997$ & $3^{\prime} 000$ \\
\hline Meyrin & $384^{\prime} 288$ & $15^{\prime} 000$ & $398^{\prime} 920$ & $30^{\prime} 000$ \\
\hline Vernier & $240^{\prime} 230$ & - & $235^{\prime} 150$ & \\
\hline Le Grand-Saconnex & $149^{\prime} 669$ & $60 ’ 834$ & $10^{\prime} 000$ & $150 \prime 938$ \\
\hline Cologny & $149^{\prime} 122$ & $10^{\prime} 053$ & $355^{\prime} 465$ & 7'168 \\
\hline Bernex & $118^{\prime} 043$ & - & $177^{\prime} 669$ & $16^{\prime} 000$ \\
\hline Versoix & $115^{\prime} 000$ & $10^{\prime} 000$ & $112 ' 154$ & - \\
\hline Plan-les-Ouates & - & - & $183^{\prime} 000$ & \\
\hline Collonge-Bellerive & - & - & $148^{\prime} 400$ & $7^{\prime} 000$ \\
\hline Grisons & $41^{\prime} 450$ & - & $41^{\prime} 920$ & $7^{\prime} 000$ \\
\hline Vaz/Obervaz & $20^{\prime} 850$ & - & $13^{\prime} 500$ & $7^{\prime} 000$ \\
\hline Davos & $20^{\prime} 600$ & - & $3 \prime 600$ & \\
\hline Jura & $13^{\prime} 000$ & $2^{\prime} 500$ & $15^{\prime} 490$ & $22^{\prime} 000$ \\
\hline Delémont & $5^{\prime} 500$ & 1'500 & $6^{\prime} 860$ & \\
\hline La Chaux-des-Breuleux & - & - & $7^{\prime} 530$ & 2000 \\
\hline Lucerne & $152^{\prime} 250$ & $3^{\prime} 000$ & $167 ' 400$ & - \\
\hline Ville de Lucerne & $922^{\prime} 000$ & $1^{\prime} 000$ & $94^{\prime} 500$ & - \\
\hline Neuchâtel & $19^{\prime} 890$ & 500 & $143^{\prime} 390$ & $33^{\prime} 000$ \\
\hline Ville de Neuchâtel & - & - & $93^{\prime} 000$ & \\
\hline Corcelles-Cormondrèche & $10^{\prime} 000$ & - & $10^{\prime} 000$ & $22^{\prime} 500$ \\
\hline La Chaux-de-Fonds & $6^{\prime} 000$ & - & $27^{\prime} 000$ & \\
\hline La Côte-aux-Fées & $2^{\prime} 000$ & 500 & $2^{\prime} 000$ & 500 \\
\hline Schaffhouse & $63^{\prime} 550$ & - & $84^{\prime} 700$ & $8^{\prime} 000$ \\
\hline Ville de Schaffhouse & $588^{\prime} 000$ & - & $75^{\prime} 000$ & $8^{\prime} 000$ \\
\hline Schwyz & $46^{\prime} 500$ & - & $83^{\prime} 000$ & $13^{\prime} 000$ \\
\hline Freienbach & $46^{\prime} 500$ & - & $73^{\prime} 000$ & $8^{\prime} 000$ \\
\hline
\end{tabular}




\begin{tabular}{|c|c|c|c|c|}
\hline \multirow[b]{2}{*}{ Cantons / Communes } & \multicolumn{2}{|c|}{2004} & \multicolumn{2}{|c|}{2003} \\
\hline & APD & AP & APD & $\mathbf{A P}$ \\
\hline Soleure & $139^{\prime} 300$ & $14^{\prime} 000$ & $63^{\prime} 120$ & $5^{\prime} 000$ \\
\hline Olten & $77^{\prime} 000$ & - & - & \\
\hline Ville de Soleure & $22^{\prime} 000$ & $6^{\prime} 000$ & - & \\
\hline Biberist & $20^{\prime} 000$ & $8^{\prime} 000$ & $17^{\prime} 000$ & $5^{\prime} 000$ \\
\hline Saint-Gall & $495^{\prime} 263$ & $11^{\prime} 000$ & $332 ' 490$ & $110^{\prime} 948$ \\
\hline Ville de Saint-Gall & $185^{\prime} 000$ & - & $1944^{\prime} 000$ & \\
\hline Kirchberg & $170^{\prime} 000$ & - & - & \\
\hline Rapperswil & $84^{\prime} 100$ & - & $61 ' 000$ & \\
\hline Mörschwil & 5000 & $10^{\prime} 000$ & - & $10^{\prime} 000$ \\
\hline Wil & - & - & - & $95^{\prime} 000$ \\
\hline Tessin & $49^{\prime} 850$ & $10^{\prime} 050$ & $106^{\prime} 850$ & $1 ' 150$ \\
\hline Lugano & - & - & $52^{\prime} 800$ & $1^{\prime} 000$ \\
\hline Bioggio & $31^{\prime} 000$ & $9^{\prime} 000$ & $35^{\prime} 000$ & \\
\hline Thurgovie & $131^{\prime} 000$ & 550 & $112^{\prime} 100$ & $10^{\prime} 000$ \\
\hline Frauenfeld & $80^{\prime} 000$ & - & $69^{\prime} 000$ & $10^{\prime} 000$ \\
\hline Kreuzlingen & $35^{\prime} 400$ & 550 & $37 ' 250$ & - \\
\hline Uri & $1 ' 500$ & $2 \prime 000$ & $5^{\prime} 000$ & - \\
\hline Altdorf & $1^{\prime} 500$ & $2 \prime 000$ & 5000 & \\
\hline Valais & $170 ' 825$ & $12^{\prime} 500$ & $78^{\prime} 690$ & $21^{\prime} 500$ \\
\hline Brig-Glis & $64^{\prime} 000$ & $2 ' 000$ & $9^{\prime} 540$ & \\
\hline Sierre & $50^{\prime} 000$ & - & $25^{\prime} 000$ & \\
\hline Sion & $25^{\prime} 375$ & $10^{\prime} 500$ & 17 '000 & $11^{\prime} 500$ \\
\hline Vaud & $400^{\prime} 010$ & $28^{\prime} 500$ & $340^{\prime} 293$ & $62 ' 315$ \\
\hline Ville de Lausanne & $178^{\prime} 150$ & $14^{\prime} 500$ & $164^{\prime} 453$ & $15^{\prime} 983$ \\
\hline Nyon & $43^{\prime} 000$ & - & $12^{\prime} 000$ & \\
\hline Vevey & $35^{\prime} 000$ & - & $35^{\prime} 000$ & \\
\hline Gland & $33^{\prime} 000$ & - & $3{ }^{\prime} 000$ & 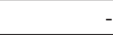 \\
\hline Morges & $1^{\prime} 500$ & $9^{\prime} 000$ & 1'500 & $17^{\prime} 648$ \\
\hline Zoug & $527^{\prime} 130$ & $9^{\prime} 860$ & $397 ' 900$ & $59^{\prime} 500$ \\
\hline Ville de Zoug & $317^{\prime} 000$ & $7^{\prime} 000$ & $223^{\prime} 900$ & $41^{\prime} 300$ \\
\hline Baar & $150^{\prime} 000$ & - & 137 '000 & $18^{\prime} 000$ \\
\hline Zurich & 2'385'776 & $118^{\prime} 677$ & 1'943'036 & $272^{\prime} 461$ \\
\hline Ville de Zurich & $943^{\prime} 786$ & - & $500^{\prime} 000$ & \\
\hline Küsnacht & $312^{\prime} 000$ & $28^{\prime} 477$ & $230 \prime 000$ & $33^{\prime} 301$ \\
\hline Illnau-Effretikon & $143^{\prime} 000$ & - & - & - \\
\hline Winterthur & $138^{\prime} 000$ & - & $139^{\prime} 000$ & \\
\hline Kloten & $135^{\prime} 000$ & - & $115^{\prime} 030$ & - \\
\hline Maur & $115^{\prime} 000$ & - & $125^{\prime} 000$ & 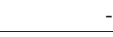 \\
\hline Stäfa & $100^{\prime} 000$ & - & - & \\
\hline Meilen & $74^{\prime} 500$ & $24^{\prime} 500$ & $125^{\prime} 000$ & $8^{\prime} 000$ \\
\hline Wetzikon (ZH) & $37^{\prime} 200$ & - & $46^{\prime} 900$ & $79^{\prime} 251$ \\
\hline Total & $12^{\prime} 665^{\prime} 665$ & $769^{\prime} 908$ & 11'614'544 & 1'300'915 \\
\hline
\end{tabular}

Source:DDC, Aide de la Suisse aux pays en développement et aux pays en transition. Statistiques 2004, Berne, mars 2006.

Remarque: Les montants figurant en regard de chaque canton indiquent le total de l'aide des communes du canton qui ont répondu à l'enquête annuelle menée par l'IUED sur mandat de la DDC.

-: montant nul.

$\mathrm{APD}=$ Aide publique au développement aux pays et territoires en développement.

$\mathrm{AP}=$ Aide publique aux pays et territoires en transition (pays de l'Europe centrale et orientale, nouveaux Etats indépendants de l'exUnion soviétique, pays et territoires en développement plus avancés). 
Le graphique 8 montre l'évolution de l'aide privée des ONG des pays du CAD depuis vingt ans en faveur des pays en développement et depuis quatorze ans en faveur des pays en transition. Il met en lumière une augmentation beaucoup plus importante de l'aide privée des ONG aux pays en développement (facteur 4,6 sur vingt ans) que celle de l'APD (facteur 2,7 sur la même période).

En ce qui concerne l'aide aux pays en transition, les facteurs sont respectivement de 14,7 et 1,2 entre 1991 et 2004.

\section{Graphique 8: «Vingt ans de coopération » - Evolution de l'aide privée des ONG des pays du CAD aux pays en développement et aux pays en transition, 1985-2004 (en millions de dollars)}

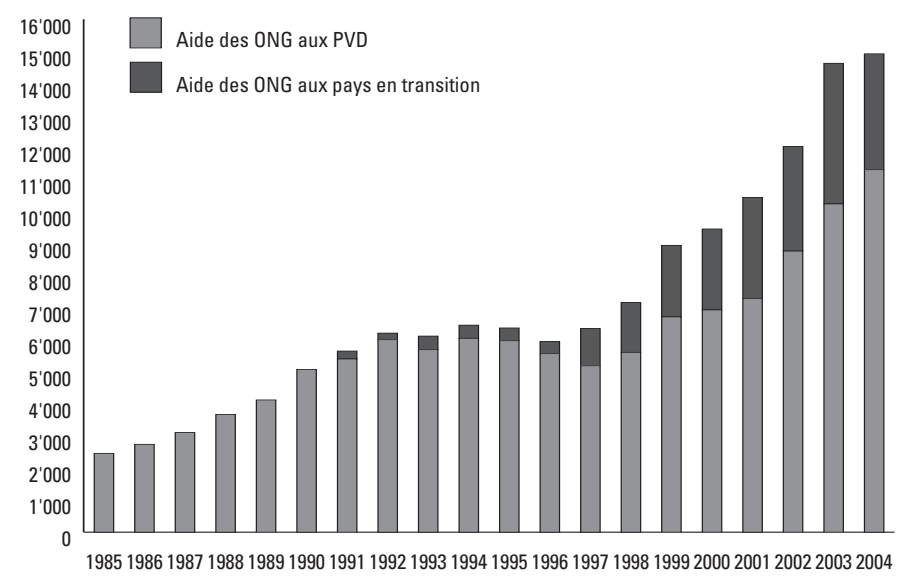

Source: OCDE, statistiques du CAD, <www.oecd.org/cad $>$.

Le graphique 9 fournit les mêmes chiffres que le graphique 8 mais pour les ONG suisses seulement. Entre 1985 et 2004, l'aide privée des ONG suisses aux pays en développement n'a cependant pas augmenté aussi vite que l'APD de la Suisse (facteur 2,2 pour l'aide des ONG suisses et facteur 2,8 pour l'APD). Il en va de même de l'aide privée des ONG suisses aux pays en transition, qui a certes augmenté, mais moins fortement que l'AP de la Suisse entre 1990 et 2004 (facteur 2,8 et facteur 7,3 respectivement). 


\section{Graphique 9: «Vingt ans de coopération » - Evolution de l'aide privée des ONG suisses aux pays en développement et aux pays en transition, 1985-2004 (en millions de francs)}

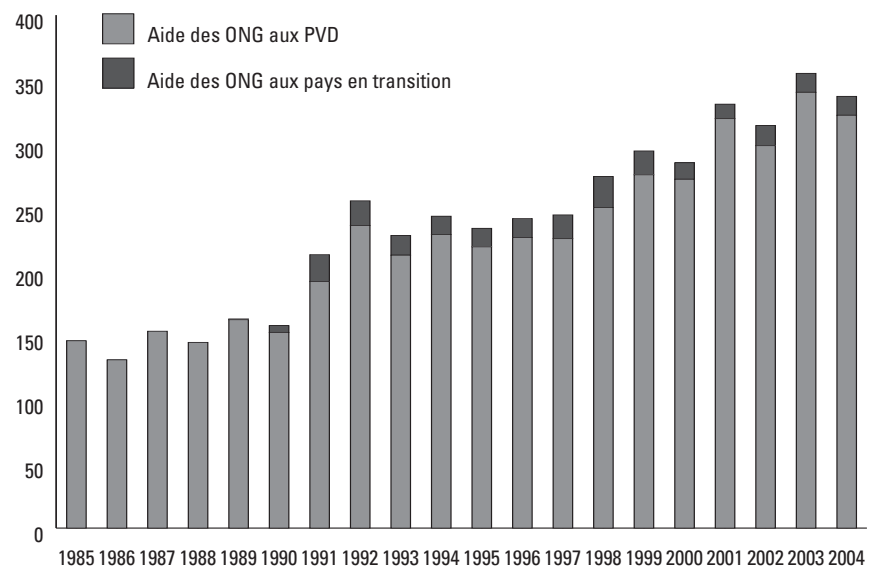

Source: DDC, Aide de la Suisse aux pays en développement et aux pays en transition. Statistiques 2004, Berne, DDC, 2006 (et éditions annuelles précédentes depuis 2001).

Les données sur l'aide privée des ONG suisses concernent les montants des projets des ONG financés par leurs fonds propres (cotisations et dons de membres, produits de récoltes de fonds, autofinancement, produits de ventes). Ne figurent dans les graphiques 8 et 9 et le tableau 9 que les montants issus de dons privés affectés par les ONG pour financer des projets de coopération ou des actions humanitaires dans les pays en développement ou en transition. Outre les 336,7 millions de francs de fonds privés récoltés par les ONG, celles-ci ont aussi obtenu des contributions de la Confédération, des cantons et communes pour la gestion de projets, pour un montant total de 208,2 millions de francs en 2004.

Le montant de l'aide privée des ONG (336,7 millions) ne correspond donc pas à leur «chiffre d'affaires » global, car il ne comprend pas les éléments suivants :

- les projets financés grâce à des contributions publiques ;

๑ les dépenses des ONG pour des projets en Suisse;

- les dépenses pour la sensibilisation et l'éducation au développement en Suisse;

$\checkmark$ les dépenses administratives générales;

- les frais de marketing et les frais liés aux campagnes de collectes de fonds.

Le tableau 11 recense les dons privés des principales ONG suisses. Il existe en Suisse quelques grandes ONG et une multitude de petites organisations. Les 35 plus grandes ONG se partagent $80 \%$ des fonds privés pour des projets. 
Tableau 11: Versements d'aide privée des principales ONG suisses, 2004 (en millions de francs et en pourcentage)

\begin{tabular}{|c|c|c|c|c|}
\hline Organisation & Aide PVD & Aide transition & $\Sigma \%^{\mathrm{a}}$ & Total \\
\hline Caritas - Suisse & $30^{\prime} 990$ & $1 ' 665$ & 9.7 & $322^{\prime} 655$ \\
\hline World Vision Schweiz & $21 ' 233$ & 447 & 16.1 & $21^{\prime} 680$ \\
\hline Fondation Hôpital Kantha Bopha Cambodge & $21^{\prime \prime 107}$ & & 22.4 & $21 ' 107$ \\
\hline Fondation Terre des hommes & $18^{\prime} 555$ & 260 & 28.0 & $18^{\prime} 815$ \\
\hline Croix Rouge Suisse CRS/SRK & $16^{\prime} 326$ & $2 ' 238$ & 33.5 & $18^{\prime} 564$ \\
\hline Entraide protestante EPER/HEKS & 14 '856 & 1'692 & 38.4 & $16^{\prime} 548$ \\
\hline MSF Médecins sans frontières & $14^{\prime} 667$ & 170 & 42.8 & $14^{\prime} 837$ \\
\hline Helvetas & $14^{\prime} 124$ & & 47.0 & $14^{\prime} 124$ \\
\hline Schweiz. Freunde der SOS Kinderdörfer & $12^{\prime} 072$ & 758 & 50.8 & $12^{\prime} 830$ \\
\hline Medair & $10 \prime 577$ & & 54.0 & $10^{\prime} 577$ \\
\hline Kinderhilfe Bethlehem & 8'932 & 80 & 56.7 & $9^{\prime} 012$ \\
\hline Action de carême - Fastenopfer & 7'452 & & 58.9 & 7'452 \\
\hline Comité suisse pour l'UNICEF & $6 ' 845$ & 170 & 61.0 & 7'015 \\
\hline OSEO/SAH & 4'686 & & 62.3 & 4'686 \\
\hline Aide aux lépreux Emmaüs & 37754 & & 63.5 & $3^{\prime} 754$ \\
\hline WWF Suisse & 3258 & 380 & 64.5 & $3^{\prime} 638$ \\
\hline Brot für Alle, Pain pour le prochain & $3{ }^{\prime} 515$ & & 65.6 & $3{ }^{\prime} 515$ \\
\hline Stif. Kinderdorf Pestalozzi & 1'789 & 1'589 & 66.6 & $3^{\prime} 378$ \\
\hline MFM & $3 \prime 072$ & & 67.5 & $3^{\prime} 072$ \\
\hline Fondation Limmat & $3{ }^{\prime} 071$ & & 68.4 & $3^{\prime} 071$ \\
\hline Mission 21 & $2 ' 967$ & 33 & 69.3 & $3^{\prime} 000$ \\
\hline Nouvelle Planète & 2976 & & 70.2 & 2,976 \\
\hline Swissaid & $2{ }^{\prime} 853$ & & 71.0 & 2,853 \\
\hline VSF & $2 ' 835$ & & 71.9 & 2,835 \\
\hline ROKPA International & 2756 & & 72.7 & 27756 \\
\hline Christoffel Blindenmission & $2 ' 650$ & 42 & 73.5 & $2{ }^{\prime} 692$ \\
\hline Seraphisches Liebeswerk & 2'194 & 317 & 74.2 & $2{ }^{\prime} 511$ \\
\hline Swisscontact & $2 ' 366$ & 144 & 75.0 & 2,510 \\
\hline Bethlehem Mission Immensee & 2'343 & 141 & 75.7 & $2 ' 484$ \\
\hline Armée du Salut - Heilsarmee & 2'378 & & 76.4 & 2378 \\
\hline Vereinigung Don Bosco Werk & $2 \prime 090$ & 164 & 77.1 & 2254 \\
\hline Fondation pro victimis & 1'985 & 221 & 77.8 & 2206 \\
\hline Weltkinderdörfer der Schwester Maria & $2 ' 200$ & & 78.4 & 2200 \\
\hline Mission der St Anna Schwestern & $2 ' 128$ & & 79.0 & $2 ' 128$ \\
\hline Mission am Nil & $2 ' 046$ & & 79.7 & 2046 \\
\hline Total des 35 organisations ci dessus & $257 ' 648$ & $9^{\prime} 482$ & 79.3 & $267^{\prime} 130$ \\
\hline 215 autres organisations & $64^{\prime} 227$ & $5^{\prime} 296$ & 20.7 & $69^{\prime} 523$ \\
\hline Total & $321 ' 875$ & $14 ' 778$ & 100.0 & $3366^{\prime} 653$ \\
\hline
\end{tabular}

Source:DDC, Aide de la Suisse aux pays en développement et aux pays en transition. Statistiques 2004, Berne, DDC, 2006.

a Pourcentage cumulé des principales ONG suisses dans le total de l'aide privée. 
Le tableau 12 permet de comparer, pour 2004, les pays qui reçoivent les montants les plus élevés d'aide publique bilatérale de la Confédération et ceux qui reçoivent le plus d'aide privée des ONG. Les classements ne sont pas très différents. Parmi les 30 pays où les ONG sont les plus présentes avec des projets financés par leurs fonds propres, 17 pays figurent aussi dans la liste des principaux bénéficiaires de l'aide publique bilatérale de la Confédération.

Tableau 12: Répartition géographique de l'aide privée et de l'aide bilatérale publique 2004 (en millions de francs et en pourcentage)

\begin{tabular}{|c|c|c|c|c|c|}
\hline \multicolumn{3}{|c|}{ Aide privée des ONG (fonds propres) } & \multicolumn{3}{|c|}{ Aide publique bilatérale Confédération } \\
\hline & $\begin{array}{c}\text { mio. } \\
\text { fr. }\end{array}$ & $\begin{array}{c}\% \\
\text { du total }\end{array}$ & & $\begin{array}{c}\text { mio. } \\
\text { fr. }\end{array}$ & $\begin{array}{r}\% \\
\text { du total }\end{array}$ \\
\hline 1. Cambodge & 22.8 & 6.8 & 1. Serbie-et-Monténégro & 70.0 & 4.7 \\
\hline 2. Inde & 16.0 & 4.8 & 2. Inde & 35.7 & 2.4 \\
\hline 3, Soudan & 11.8 & 3.5 & 3. Mozambique & 34.4 & 2.3 \\
\hline 4. Zones admin. palestini & רe 11.6 & 3.4 & 4. Tanzanie & 32.4 & 2.2 \\
\hline 5. Serbie-et-Monténégro & 11.1 & 3.3 & 5. Bolivie & 26.0 & 1.8 \\
\hline 6. Ethiopie & 8.5 & 7.4 & 6. Burkina Faso & 25.4 & 1.7 \\
\hline 7. Brésil & 8.3 & 2.5 & 7. Pérou & 24.9 & 1.7 \\
\hline 8. Bosnie-Herzégovine & 7.9 & 2.3 & 8. Vietnam & 24.5 & 1.7 \\
\hline 9. Afghanistan & 7.2 & 2.1 & 9. Afghanistan & 23.4 & 1.6 \\
\hline 10. Tanzanie & 6.2 & 1.9 & 10. Nicaragua & 21.8 & 1.5 \\
\hline 11. Iran & 6.2 & 1.8 & 11. Tadjikistan & 21.2 & 1.4 \\
\hline 12. Burkina Faso & 6.0 & 1.8 & 12. Népal & 19.4 & 1.3 \\
\hline 13. Colombie & 5.7 & 1.7 & 13. Madagascar & 17.7 & 1.2 \\
\hline 14. Haïti & 5.5 & 1.6 & 14. Tchad & 17.5 & 1.2 \\
\hline 15. Bangladesh & 5.5 & 1.6 & 15. Soudan & 16.8 & 1.1 \\
\hline 16. Mali & 5.3 & 1.6 & 16. Zones admin. palestinienne & 16.7 & 1.1 \\
\hline 17. Mozambique & 5.3 & 1.6 & 17. Russie & 16.1 & 1.1 \\
\hline 18. Bolivie & 5.3 & 1.6 & 18. Pakistan & 15.9 & 1.1 \\
\hline 19. Congo, Rép. dém. & 5.2 & 1.5 & 19. Bosnie-Herzégovine & 15.8 & 1.1 \\
\hline 20. Nicaragua & 5.1 & 1.5 & 20. Macédoine (ERYM) & 15.6 & 1.1 \\
\hline 21. Ouganda & 4.6 & 1.4 & 21. Bangladesh & 14.7 & 1.0 \\
\hline 22. Pérou & 4.6 & 1.4 & 22. Bulgarie & 13.5 & 0.9 \\
\hline 23. Caméroun & 4.6 & 1.4 & 23. Kirghize, Rép. & 13.0 & 0.9 \\
\hline 24. Vietnam & 4.6 & 1.4 & 24. Ouzbékistan & 12.3 & 0.8 \\
\hline 25. Philippines & 4.4 & 1.3 & 25. Roumanie & 11.9 & 0.8 \\
\hline 26. Albanie & 4.2 & 1.3 & 26. Bénin & 11.7 & 0.8 \\
\hline 27. Guatemala & 4.1 & 1.2 & 27. Mali & 11.1 & 0.8 \\
\hline 28. Libéria & 4.0 & 1.2 & 28. Colombie & 10.2 & 0.7 \\
\hline 29. Kirghize, Rép. & 3.8 & 1.1 & 29. Géorgie & 10.0 & 0.7 \\
\hline 30. Rép. tchèque & 3.8 & 1.1 & 30. Niger & 9.6 & 0.7 \\
\hline Total pays ci-dessus & 209.4 & 62.2 & Total pays ci-dessus & 609.0 & 41.3 \\
\hline Autres pays & 127.3 & 37.8 & Autres pays & 866.5 & 58.7 \\
\hline & & & & & 0.0 \\
\hline Total de l'aide ONG & 336.7 & 100.0 & Total aide bilatérale & 1475.5 & 100.0 \\
\hline
\end{tabular}

Sources: DDC, seco, Rapport annuel de la coopération internationale de la Suisse 2004, Berne, 2005; DDC, Aide de la Suisse aux pays en développement et aux pays en transition. Statistiques 2004, Berne, DDC, 2006.

Remarque: Les pays en gras sont ceux qui se retrouvent dans les deux listes. 